\title{
Learning curve for collective behavior of zero-intelligence agents in successive job-hunting processes with a diversity of Jaynes-Shannon's MaxEnt principle
}

\author{
He Chen* and Jun-ichi Inoue ${ }^{\dagger}$ \\ Graduate School of Information Science and Technology \\ Hokkaido University, N14-W9, Kita-ku, Sapporo 060-0814, Japan
}

August 10, 2021

\begin{abstract}
Inspired by the unsupervised learning or self-organization in the machine learning context, here we attempt to draw 'learning curve' for the collective behavior of job-seeking 'zero-intelligence' labors in successive job-hunting processes. Our labor market is supposed to be opened especially for university graduates in Japan, where the students have several successive chances $n=0,1,2, \cdots$ to obtain their positions within an academic (business) year. In this sense, the 'cumulative unemployment rate' in our model system is regarded as an error-measurement in the collective intelligence of students, and the job-hunting stage $n$-dependence of the error constructs a learning curve. In our simple toy-model of probabilistic labor market, the diversity of students' behavior is built-in by means of the JaynesShannon's MaxEnt (Maximum Entropy) principle. Then, we discuss the speed of convergence for the error-measurement, where we consider a scenario in which the students do not use any information about the result of job-hunting processes in the previous stage. Our approach enables us to examine the existence of the condition on which macroscopic quantity, say, 'stage-wise unemployment rate' becomes 'scale-invariant' in the sense that it does not depend on the job-hunting stage $n$. From the macroscopic view point, the problem could be regarded as a human resource allocation.
\end{abstract}

Keywords: Labor market, Microscopic description of macroeconomics, Learning curve, Statistical mechanics, Jaynes-Shannon's MaxEnt principle, Agent-based modeling, Econophysics

JEL Codes: J42, E24, C51

\section{Introduction}

Deterioration of the employment rate is now one of the most worrying problems in Japan METI, MHLW, Recruit Works Institute. Actually, various attempts to overcome these difficulties have been done by governments. Apparently, labor (work) is important not only for each of us to earn our daily bread, but also for our state to keep the revenues by collecting the taxes from labors. Especially, in recent Japan, the employment rate is getting worse and the government has over-issued quite a lot of government bonds to compensate a lack of the tax revenues and the national debt is now becoming a serious risk to cause a national-wide bankruptcy.

To consider the effective policy and to carry out it for sweeping away the unemployment uncertainty caused by the so-called 'mismatch', it seems that we should investigate the labor markets scientifically and if possible, one should simulate artificial labor markets in computer to reveal the essential features of the problem. In fact, in macroeconomics (labor science), there exist a lot of effective attempts to discuss the macroscopic properties Aoki and Yoshikawa (2006), Boeri and Ours (2008), Gabriele (2002), Fagiolo, Dosi and Gabriele (2004), Neugart (2004)]. However, apparently, the macroscopic approaches lack of their microscopic view points, namely, in their arguments, the behavior of microscopic heterogeneous agents such as labors or companies are neglected.

In our preliminary studies Hino and Inoue (2011)], we attempted to construct such microscopic models of probabilistic labor markets in which the labors (job-seekers) and the companies behave probabilistically. Then, we evaluated the unemployment and the inflation rates as relevant macroscopic

*Email: chen@complex.ist.hokudai.ac.jp

$\dagger$ Email: jinoue@cb4.so-net.ne.jp

$\ddagger$ This manuscript was accepted by Evolutionary and Institutional Economics Review. 
quantities and draw the so-called Philips curves [Philips (1958)], which have a universal fitting curve: (inflation rate $)=(\text { unemployment rate })^{c}+b, b, c<0$. However, in the model, we introduced a lot of parameters to describe the labor market and it seems not to be far-sighted to reveal the essential properties of the market and we need much more simpler or 'minimal' models to discuss the macroscopic behaviour from the microscopic descriptions.

Taking this fact in mind, in the references Chen and Inoue (2012a), Chen and Inoue (2012b)] we proposed a simple probabilistic model based on the concept of statistical mechanics for probabilistic labor markets, in particular, Japanese labor markets for university (college) graduates. In this agentbased modeling, we introduced the energy function for each company. The energy function describes the power of each company to gather the entry sheets (application letters) of the students. The energy consists of two distinct factors, namely, ranking preference and market history effects. The later acts as 'negative feedback' on the ranking preference. Then, we evaluated the relevant macroscopic quantity, namely, unemployment rate by means of the microscopic variables. We also drew the socalled Beveridge curve (UV curve: 'Unemployment -Vacancy') which shows the relationship between the unemployment rate and the number of vacancies (job-offer ratio). We found that the system undergoes a phase transition with spontaneous symmetry breaking from the good employment rate phase to the poor employment rate phase when one increases the degree of ranking preference. In the references [Chen and Inoue (2012a), Chen and Inoue (2012b)], we also utilized the macroscopic Neugart model [Neugart (2004)] which describes the Philips curve as an attractor of chaotic maps. We used the update equation for the inflation rate accompanying with our probabilistic model to derive the Philips curve for our model system. We found that the Philips curve is actually obtained by the 'mixture model'.

In Japanese labor markets for university graduates, the students have several successive chances to obtain their positions within an academic (business) year. After each opportunity (stage) is over, the numbers of both students and positions (namely, the so-called 'effective system size') are reduced remarkably because several excellent students get their job immediately after they apply for. Accordingly, the macroscopic quantities such as unemployment rate $U$, labor shortage ratio $\Omega$ or job offer ratio $\alpha$ changes as $U^{(0)} \rightarrow \cdots U^{(n)}, \Omega^{(0)} \rightarrow \cdots \Omega^{(n)}$ or $\alpha^{(0)} \rightarrow \cdots \alpha^{(n)}$. However, it is highly non-trivial whether some of them could be scale invariant or not, that is to say, it is not easy for us to conclude that there exists the condition on which a macroscopic quantity, say, $U^{(n)}$ satisfies $U^{(n)}=$ constant for any jobhunting stage $n$. Here we shall focus on this issue and clarify the $n$-dependence (or independence) of Japanese labor markets for university graduates by making use of both extensive computer simulations and empirical data analysis. In order to investigate these aspects of labor markets, here we attempt to draw 'learning curve' for the collective behavior of job-seeking 'zero-intelligence' labors in successive job-hunting process. Our labor market is supposed to be opened especially for university graduates in Japan, where the students have several successive chances $n=0,1,2, \cdots$ to obtain their positions within an academic (business) year. In this sense, the 'cumulative unemployment rate' in our model system is regarded as an error-measurement in the collective intelligence of students and the job-hunting stage $n$-dependence of the error constructs a learning curve. In our toy-model of probabilistic labor market, the diversity of students' behavior is built-in by means of the Jaynes-Shannon's MaxEnt (Maximum Entropy) principle. We discuss the speed of convergence for the error measurement, where we consider a scenario in which the students do not use any information about the result of job-hunting processes in the previous stages.

This paper is organized as follows. In the next section 2 we introduce several important concepts used in this paper. In particular, we account for two distinct quantities, namely, 'cumulative' and 'stagewise' quantities. The generic properties of them would be explained. In the same section, we also show several important empirical findings. In section 3 we introduce our probabilistic labor market which is constructed by means of Jaynes-Shannon's MaxEnt principle. The numerical results are given in the next section 4. The last section is devoted to summary.

\section{Empirical evidence}

As we already mentioned in the previous section, employment uncertainty in young generation, especially in university graduates has been one of the serious social problems in Japan. Actually, the employment rate (the informal acceptance rate) reported in four times (October, December and February, April in the next year) within a business year is getting worse. In Figure 1(left), we plot the empirical trends of employment rate $1-U$ changing in year for the four employment opportunities (let us specify the stage by $n$ as $n=0,1,2$ and 3 ) in the last seventeen years. From this panel, we clearly observe that in each year the employment rate increases monotonically as the stage $n$ goes on (see the right panel). This means that the students and companies make up these four labor markets having the different (reduced) system sizes. Namely, after the first $n=0$ stage, several excellent students go away from the market and several 

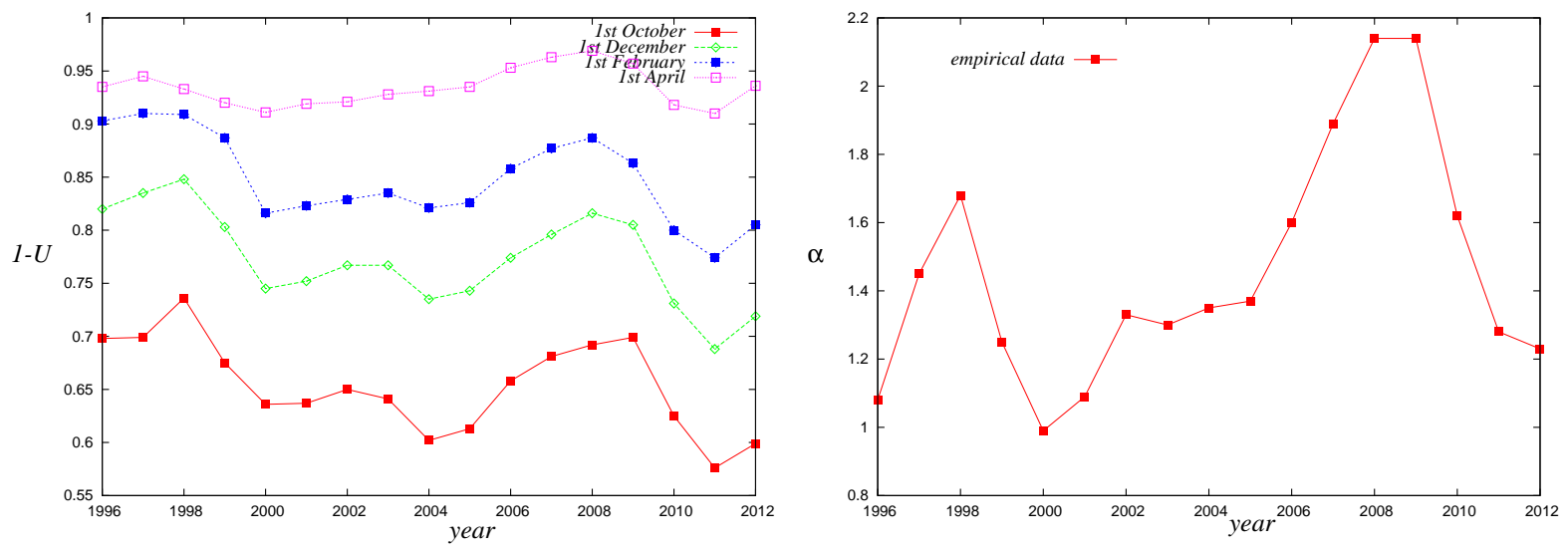

Figure 1: The left panel shows the changing trends in cumulative employment rate for each employment opportunities, based on employment statistical data of university graduates in 1996 to 2012 by MEXT (Ministry of Education, Culture, Sports, Science and Technology) \& MHLW (Ministry of Health, Labor and Welfare) [MEXT. The right panel exhibits the annual job-offer ratio at October, that is, $\alpha^{(0)} \equiv \alpha$. We find that the $\alpha$ is greater than unity except for in 2000.

popular companies also close recruiting students because they might satisfy their quota immediately after they start to advertise for the candidates for the posts. Hence, the size of the market (the number of students and positions) is definitely getting small as the job-hunting stage goes on as $n=0,1,2, \cdots$.

In the right panel of Figure 1, we plot the annual job-offer ratio $\alpha$ which is usually released to the public in every October by MEXT (Ministry of Education, Culture, Sports, Science and Technology) \& MHLW (Ministry of Health, Labor and Welfare) [MEXT]. From this panel, we observe that the $\alpha$ is greater than 1 except for the statistics in 2000. In this sense, Japanese labor markets for university graduates are almost 'seller's market' in which the number of opened positions is larger than that of job-seeking students.

\section{1 'Stage-wise' unemployment rate and 'cumulative' employment rate}

In the previous section, we found that the employment rate $1-U$ is getting better as the term goes on as October, December, February, and April. This fact is naturally accepted because the employment rate $1-U$ is 'cumulative quantity'. In this sense, the $1-U$ is a monotonically increasing function of the term. However, when we assume that the market might shrink gradually as the term goes on, namely, the size of the market such as the number of students, positions might decrease, one could define the 'size-reduced market' which is induced from the market at the previous stage (term) after. In this sense, there might exist successive opportunities for students who failed in the previous stages to apply to the positions in companies in the size-reduced market after each term is over.

Then, an interesting query might arise in our mind. Namely, is it possible for us to conclude that the unemployment rate at the stage $n$ - what we call 'stage-wise' unemployment rate - should be the size (scale)-independent as $U^{(n)}=U(=$ constant $)$ ?, where we defined the following 'stage-wise' unemployment rate

$$
U^{(n)}=\frac{\# \text { of students who do not get any job even after the } n \text {-stage is over }}{\# \text { of students who are looking for the job after the }(n-1) \text {-stage is over }} .
$$

To answer this highly relevant question, let us define the 'cumulative' employment rate at each stage $n=1,2, \cdots$ by

$$
(1-U)_{n}=\frac{\text { total \# of students who got the position until the stage } n}{\text { total \# of students }} .
$$

Obviously, unemployment rate shown in Figure 1 (left) is the above cumulative employment rate and it increases monotonically up to $(1-U)_{n}=1$ as the stage $n$ goes on. On the other hand, the behavior of stage-wise unemployment rate $U^{(n)}$ might be more complicated and it contains useful information about the difficulties of matching between students and companies in each job-hunting process. However, unfortunately, there is no empirical data conferring the stage-wise unemployment rate opened to the public and we should estimate the quantities from the available data such as $(1-U)_{n}$.

\subsection{Global mismatch and its measurement}

We should notice that in our model system, one has three relevant quantities, namely, unemployment rate $U^{(n)}$, labor shortage ratio $\Omega^{(n)}$ and job offer ratio $\alpha^{(n)}$. To obtain a useful relationship between these 
quantities, let us define the total number of students and companies by $N$ and $K$, respectively. Each student and company are specified by labels $i=1, \cdots, N ; k=1, \cdots, K$, and let $v_{k}^{*}$ be the quota of the company $k$ and we define the number of students whom the company $k$ obtains by $m_{k}$. Then, we have

$$
\Omega=\frac{1}{V} \sum_{k=1}^{K}\left(v_{k}^{*}-m_{k}\right)=1-\frac{1}{V} \sum_{k=1}^{K} v_{k}^{*}=1-\alpha^{-1}(1-U),
$$

where we used the definitions of total vacancy

$$
V \equiv \sum_{k=1}^{K} v_{k}^{*}
$$

and the job offer ratio

$$
\alpha \equiv V / N .
$$

Apparently, for $\alpha=V / N>1$, the labor market behaves as a 'seller's market', whereas for $\alpha<1$, the market becomes a 'buyer's market'. Therefore, one confirms that $U, \Omega$ and $\alpha$ should satisfy the following single equation Chen and Inoue (2012a), Chen and Inoue (2012b).

$$
U=\alpha \Omega+1-\alpha
$$

By solving the above equation with respect to $\Omega$, we have the alternative form

$$
\Omega=\frac{\alpha-(1-U)}{\alpha} .
$$

By using this equation (77), we calculate the labor shortage ratio $\Omega$ by means of the observables $1-U$ and $\alpha$ as we showed in Figure 1. Here it should be kept in mind that the above relation is satisfied for cumulative quantities although we did not mention it clearly. Namely, (7) (or equation (6)) should be written as

$$
\Omega_{n}=\frac{\alpha-(1-U)_{n}}{\alpha}
$$

where $\Omega_{n}$ stands for the cumulative labor shortage ratio. It could be defined explicitly as

$$
\Omega_{n}=\frac{\# \text { of positions which are opened for students until the stage } n}{\text { total \# of positions in society }},
$$

which is different from the stage-wise labor shortage ratio

$$
\Omega^{(n)}=\frac{\# \text { of positions which are still opened for students even after } n \text {-stage is over }}{\# \text { of positions which are opened for students after }(n-1) \text {-stage is over }} .
$$

In Figure 2, we plot the cumulative employment rate $(1-U)_{n}$ (left) and labor shortage ratio $\Omega_{n}$ (right)
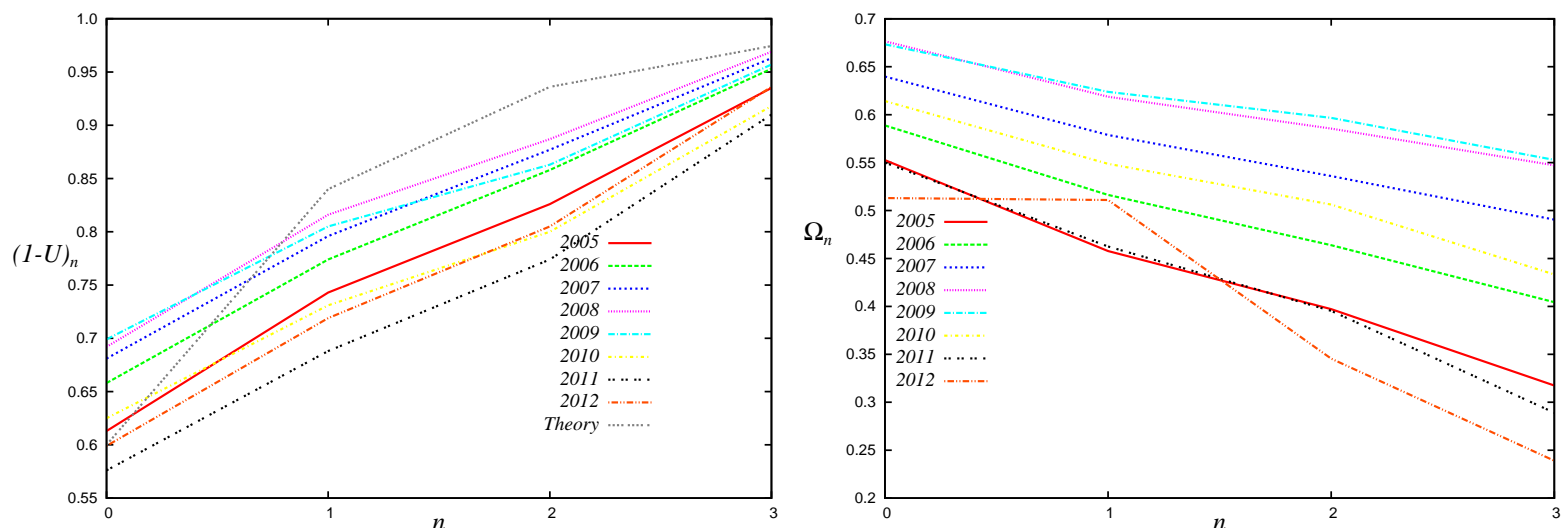

Figure 2: The cumulative employment rate $(1-U)_{n}$ (left) and labor shortage ratio $\Omega_{n}$ (right) evaluated by employment statistical data of university graduates in 1996 to 2012 by MEXT \& MHLW [MEXT]. $\Omega_{n}$ is calculated by $\Omega_{n}=\{\alpha-$ $\left.(1-U)_{n}\right\} / \alpha$. A line caption 'Theory' in the left panel denotes $(1-U)_{n}=1-U^{n}$ which is obtained from the assumption $U^{(n)}=U=0.4$. From this panel, we find that the empirical data does not follow the theoretical law: $1-U^{n}$.

evaluated by employment statistical data of university graduates in 1996 to 2012 by MEXT \& MHLW 
MEXT. From the right panel, we are confirmed that the $(1-U)_{n}$ increases monotonically as the stage $n$ gains. On the other hand, the cumulative labor shortage ratio $\Omega_{n}$ is apparently a monotonically increasing function of $n$.

In following, we write $\Omega_{n} \equiv \Omega,(1-U)_{n} \equiv 1-U$ for simplicity and draw the $U$ - $\Omega$ curve using the empirical data for the cumulative employment rate $1-U$.

In Figure 3, we show the $U-\Omega$ curves for seventeen years in October, December, February and April, which are calculated by using the data [MEXT]. From these four panels, we find that the degree of global
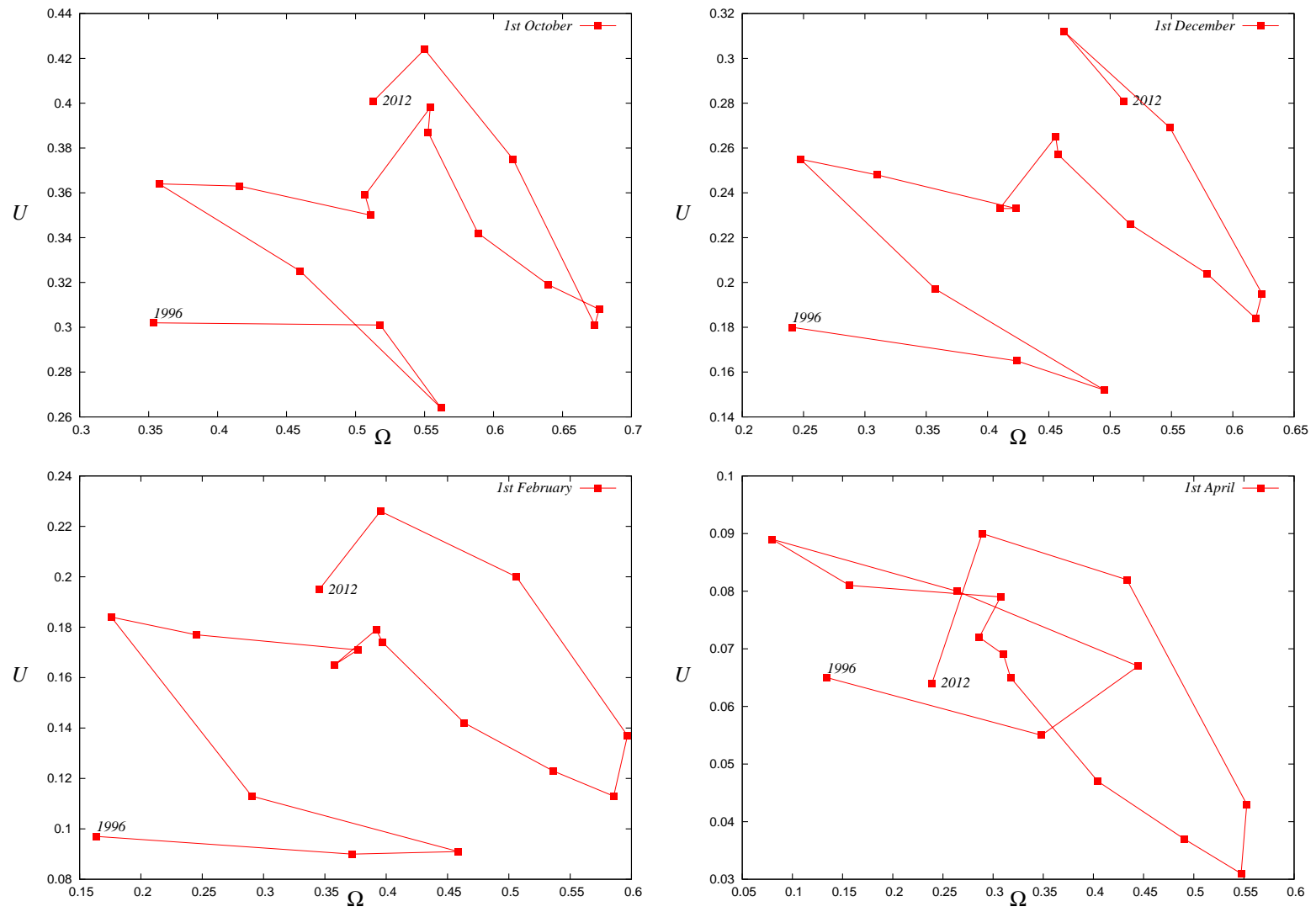

Figure 3: The $U-\Omega$ curves for October, December, February and April, which are calculated by empirical data of $1-U$ [MEXT] and equation (7). We find that the degree of global mismatch between companies and students in 2010 is the worst in last seventeen years. However, in 2011 and 2012, the mismatch is even improved by compromise between companies and students.

mismatch between companies and students in 2010 is the worst in the past seventeen years. However, in 2011 and 2012, the mismatch is even improved by compromise between companies and students.

As we have shown above, the $U-\Omega$ curve tells us useful information about the global mismatch. Especially, the trajectory as shown in Figure 3 is reflected by changing students' tendencies for the choice of companies, government's policy, or business cycle in the society.

\subsection{Representation of $\left(\alpha^{(n)}, U^{(n)}, \Omega^{(n)}\right)$ in terms of $(1-U)_{n},(1-U)_{n-1}$ and $\alpha$}

In the previous section, we showed the $U$ - $\Omega$ curve for the cumulative quantities $(1-U)_{n}$ and $\Omega_{n}$. From the result, we were confirmed that global mismatch between students and companies was actually enhanced around 2010. However, the information about the $U-\Omega$ curve does not tell us to what extent the market becomes difficult or easy for students to obtain the jobs, or to what extent the market is sufficient or insufficient for companies to fill their quota as the stage $n$ goes on. In order to investigate this kind of macroscopic properties of the market, we should draw the $U-\Omega$ curve for 'stage-wise' quantities instead of the cumulative ones.

Then, we should notice that the relationship (7) (or equation (6) ) should be satisfied for the 'stagewise' quantities $\left(\alpha^{(n)}, U^{(n)}, \Omega^{(n)}\right)$ as well. Hence, by setting $\Omega=\Omega^{(n)}, U=U^{(n)}$ and $\alpha=\alpha^{(n)}$, we obtain a single relationship:

$$
U^{(n)}=\alpha^{(n)} \Omega^{(n)}+1-\alpha^{(n)} .
$$

We should keep in mind that $\alpha^{(0)}=\alpha, U^{(0)}=U_{0}=U, \Omega^{(0)}=\Omega_{0}=\Omega$, and the stage-wise job-offer ratio 
$\alpha^{(n)}$ is defined as

$$
\alpha^{(n)}=\frac{\# \text { of positions opened for students after } n \text {-stage is over }}{\# \text { of students who are looking for the position after } n \text {-stage is over }} .
$$

From the definition, $\alpha \equiv \alpha^{(0)}$ appearing in equation (7) for the cumulative quantities should be $\alpha^{(0)}=$ $V / N$ and the counter empirical data is opened to the public in the October's statistics.

Here we attempt to rewrite the set of relevant quantities $\left(\alpha^{(n)}, U^{(n)}, \Omega^{(n)}\right)$ in the stage-wise sense at the $n$-th job-hunting stage in terms of the cumulative employment rates $(1-U)_{n},(1-U)_{n-1}$ and job-offer ratio $\alpha \equiv \alpha^{(0)}$. For this purpose, we first examine the expression for $\alpha^{(1)}$.

The total positions (vacancies) after the first stage is over, namely, $V^{(1)}$ is given by

$$
V^{(1)}=V^{(0)}-\left(1-U^{(0)}\right) N=\left\{\alpha-1+U^{(0)}\right\} N
$$

where we used $V \equiv V^{(0)}=\alpha N$. On the other hand, the number of students who could not get any position at $n=1$ is given as $N^{(1)} \equiv N U^{(0)}$. Hence, we have

$$
\alpha^{(1)}=\frac{V^{(1)}}{N^{(1)}}=\frac{\left\{\alpha-1+U^{(0)}\right\} N}{U^{(0)} N}=\frac{\alpha-1+U^{(0)}}{U^{(0)}} .
$$

Using the same way as the above $V^{(1)}$, we obtain the number of positions still remaining after the second stage as

$$
\begin{aligned}
V^{(2)} & =V^{(1)}-\left(1-U^{(1)}\right) U^{(0)} N \\
& =V-\left(1-U^{(0)}\right) N-\left(1-U^{(1)}\right) U^{(0)} N=\left\{\alpha-1+U^{(1)} U^{(0)}\right\} N .
\end{aligned}
$$

Therefore, when we notice that $N^{(2)}=U^{(1)} U^{(0)} N$, one can rewrite the $\alpha^{(2)}$ in terms of $U^{(0)}, U^{(1)}$ and $\alpha$ as follows.

$$
\alpha^{(2)}=\frac{V^{(2)}}{N^{(2)}}=\frac{\alpha-1+U^{(1)} U^{(0)}}{U^{(1)} U^{(0)}}
$$

Repeating this procedure up to $n$-stage, we easily obtain

$$
\frac{V^{(n)}}{N}=\alpha-1+\prod_{k=0}^{n-1} U^{(k)}, \frac{N^{(n)}}{N}=\prod_{k=0}^{n-1} U^{(k)}
$$

and this simply reads

$$
\alpha^{(n)}=\frac{V^{(n)}}{N^{(n)}}=\frac{\alpha-1+\prod_{k=0}^{n-1} U^{(k)}}{\prod_{k=0}^{n-1} U^{(k)}}(n \geq 1), \alpha^{(0)}=\alpha .
$$

We next rewrite the above $\alpha^{(n)}$ by means of the cumulative employment rate $(1-U)_{n-1}$ which is available as an empirical data set shown in Figure 1. Obviously, $V^{(n)}$ is given in terms of $(1-U)_{n-1}$ as $V^{(n)}=\left\{\alpha-(1-U)_{n-1}\right\} N$. On the other hand, the number of students who could not get any job until $n$-stage is evaluated by $N^{(n)}=N-(1-U)_{n-1} N=\left\{1-(1-U)_{n-1}\right\} N$. Thus, we have

$$
\alpha^{(n)}=\frac{V^{(n)}}{N^{(n)}}=\frac{\alpha-(1-U)_{n-1}}{1-(1-U)_{n-1}} .
$$

By comparing equations (18) and (19), we immediately obtain

$$
(1-U)_{n-1}=1-\prod_{k=0}^{n-1} U^{(k)} .
$$

In order to derive the expression of 'stage-wise' unemployment rate $U^{(k)}$ in terms of the cumulative employment rate $(1-U)_{k}$, we shall write down (20) for $n=2,1$ and $n=0$. This procedure immediately leads to $(1-U)_{2}=1-U^{(0)} U^{(1)} U^{(2)},(1-U)_{1}=1-U^{(0)} U^{(1)}$ and $(1-U)_{0}=1-U^{(0)}$. Solving these equations with respect to $U^{(k)}, k=0,1,2$, one obtains

$$
U^{(0)}=1-(1-U)_{0}, U^{(1)}=\frac{1-(1-U)_{1}}{1-(1-U)_{0}}, U^{(2)}=\frac{1-(1-U)_{2}}{1-(1-U)_{1}} .
$$

The above result is easily generalized to $U^{(n)}$ as

$$
U^{(n)}=\frac{1-(1-U)_{n}}{1-(1-U)_{n-1}} .
$$


It is quite easy for us to prove that $(0 \leq) U^{(n)} \leq 1$ holds. When we notice that the cumulative employment is a monotonically increasing function of $n$, one has $(1-U)_{n-1} \leq(1-U)_{n}$, that is, $1-(1-U)_{n} \leq$ $1-(1-U)_{n-1}$. Hence, we conclude

$$
(0 \leq) \frac{1-(1-U)_{n}}{1-(1-U)_{n-1}} \equiv U^{(n)} \leq 1
$$

Using the relation (11), we immediately obtain

$$
\Omega^{(n)}=\frac{U^{(n)}+\alpha^{(n)}-1}{\alpha^{(n)}}=\frac{\alpha-(1-U)_{n}}{\alpha-(1-U)_{n-1}} .
$$

From the fact that $(1-U)_{n}$ decreases monotonically as $n$ increases, we can easily show $(0 \leq) \Omega^{(n)} \leq 1$ using the same argument as in $U^{(n)}$ (the above equation (23) ).

Let us summarize the result.

$$
\left(\alpha^{(n)}, U^{(n)}, \Omega^{(n)}\right)=\left(\frac{\alpha-(1-U)_{n-1}}{1-(1-U)_{n-1}}, \frac{1-(1-U)_{n}}{1-(1-U)_{n-1}}, \frac{\alpha-(1-U)_{n}}{\alpha-(1-U)_{n-1}}\right)
$$

In following, we shall investigate the generic properties of these stage-wise quantities.

\subsubsection{On the monotonic behavior of stage-wise job-offer ratio}

It should bear in mind that the behavior of the stage-wise job-offer ratio $\alpha^{(n)}$ is strongly dependent on the initial value $\alpha^{(0)}=\alpha$. Actually, one can evaluate the difference (gap) $\alpha^{(n)}-\alpha^{(n-1)}$ as

$$
\alpha^{(n)}-\alpha^{(n-1)}=\frac{(\alpha-1)\left\{(1-U)_{n-1}-(1-U)_{n-2}\right\}}{\left\{1-(1-U)_{n-1}\right\}\left\{1-(1-U)_{n-2}\right\}} .
$$

Taking into account the the fact that the cumulative employment rate $(1-U)_{n}$ is a monotonically increasing function of stage $n$, namely, $(1-U)_{n-1} \geq(1-U)_{n-2}$, and it is lower than unity, that is, $(1-U)_{n-1},(1-U)_{n-2} \leq 1$, we conclude that

$$
\begin{aligned}
& \alpha^{(n)}>\alpha^{(n-1)} \quad(\alpha>1: \text { monotonically increasing }) \\
& \alpha^{(n)}<\alpha^{(n-1)} \quad(\alpha<1 \text { : monotonically decreasing })
\end{aligned}
$$

Apparently, the stage-wise job-offer ratio is one of the measurement to quantify the difficulties of matching process between students and companies on the condition that one does not consider the extreme 'biasedbehavior' of the students' preferences. Therefore, the above result tells us that the job-hunting becomes more difficult as stage $n$ goes on when the job-offer ratio released to the public in October $\alpha^{(0)}=\alpha$ is lower than 1, whereas it becomes easier as the stage $n$ increases when $\alpha>1$. Actually in Figure 4 , we plot the $\alpha^{(n)}$ by making use of the empirical data. The left panel in Figure 4 shows the results for 1996, 2005 and 2009 having $\alpha>1$, whereas the right panel is the result for the market in 2000 having $\alpha=0.99<1$. From this figure, we clearly find that the behavior of $\alpha^{(0)}$ is categorized into distinct two classes by $\alpha>1$ or $\alpha<1$. It should be stressed that this result is independent on the choice of concrete matching algorithm for collective intelligence of students in job-hunting process. We should mention that no similar monotonic behavior is observed in $\Omega^{(n)}$ or $U^{(n)}$.

From the above general result, we obtain several insights into the behavior of recent Japanese labor market for university graduates. Very recently, in April 2013, a Japanese think tank, Recruit Works Institute Recruit Works Institute (2013) reported that the job-offer ratio in 2012 for the market at the first stage which includes all under-, graduate students and all companies was $\alpha^{(0)} \equiv \alpha=1.28(>1)$. Hence, from the above discussion, the stage-wise job-offer ratio $\alpha^{(n)}$ increases as $n$ increases. Thus, the students should not rush themselves to get the positions because it becomes much easier for them to obtain the jobs as $n$ goes on. However, the think tank also reported that the 'effective' job-offer ratio was $\alpha=0.54(<1)$ when we restrict ourselves to the market of established and large-scale companies having more than 5000 employees. This fact implies that the students who are looking for the positions in such established companies should hurry to obtain the jobs at as early-stage as possible because the stage-wise $\alpha^{(n)}$ decreases monotonically to zero for $\alpha<1$. On the other hand, the effective job-offer ratio for the market in which there are only small enterprises having less than 300 employees was 3.26 in 2012 . This fact might be a reason why a global mismatch is difficult to be overcame in recent Japanese labor markets for university graduates. 

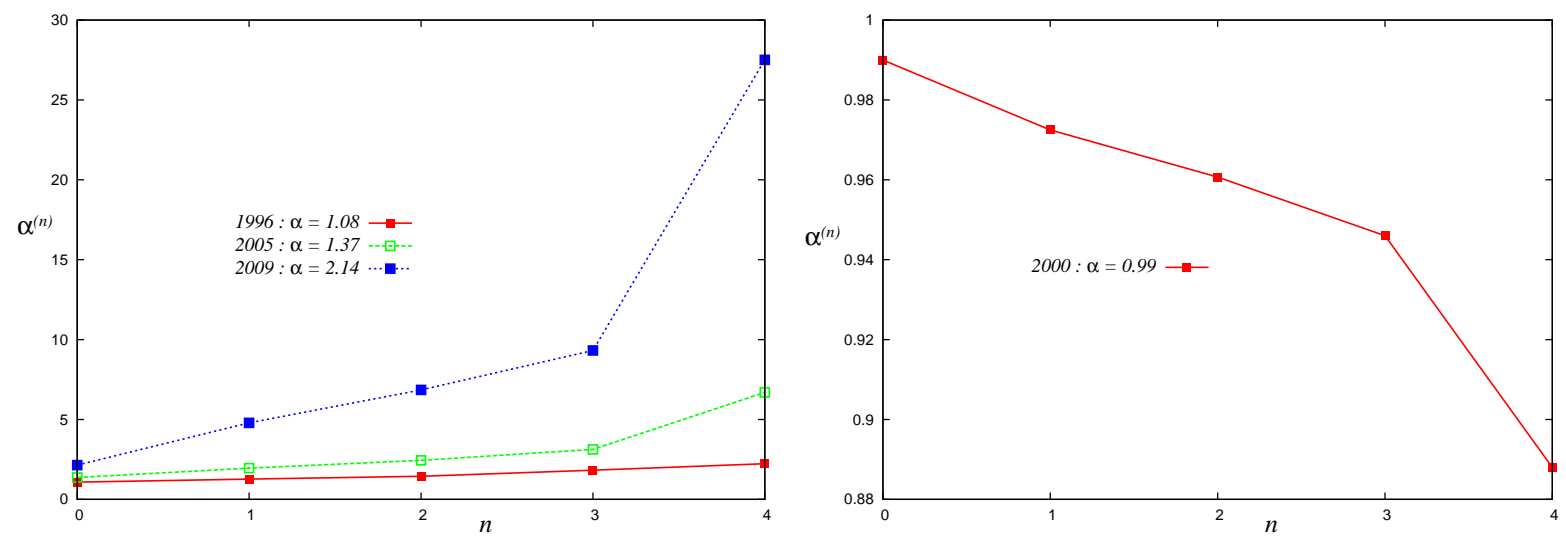

Figure 4: The stage-wise job-offer ratio $\alpha^{(n)}=\left\{\alpha-(1-U)_{n-1}\right\} /\left\{1-(1-U)_{n-1}\right\}$. The right panel shows several typical examples for the case of $\alpha>1$ (for the statistics in 1996, 2005 and 2009), whereas the right panel is shown as a result for $\alpha<1$ (for the statistics in 2000).

\subsubsection{The asymptotic limit}

As we already explained in the introduction, in Japan, the statistics for the cumulative employment rate is opened to the public in four seasons, namely, in October $(n=0)$, December $(n=1)$, February $(n=2)$ and April $(n=3)$. However, it is possible for us to imagine the case of quite large $n$, especially, the asymptotic limit of stage-wise quantities $\left(\alpha^{(n)}, U^{(n)}, \Omega^{(n)}\right)$ in the limit of $n \rightarrow \infty$. Hence, here we shall consider the limit $n \rightarrow \infty$ in the results (25) obtained in the previous sections.

We first consider the case of $\alpha<1$. To consider the limit $n \rightarrow \infty$, we should notice that the number of students who get their jobs becomes identical to the total vacancy $V$ in the limit of $n \rightarrow \infty$ for $\alpha<1$. Namely

$$
\lim _{n \rightarrow \infty}(1-U)_{n}=\frac{V}{N}=\alpha
$$

In this sense, the cumulative employment rate could not increases up to unity and it is apparently bounded from the above by the job-offer ratio opened in October $(n=0)$, namely, $\alpha^{(0)}=\alpha$. Therefore, we easily confirm at the end

$$
\lim _{n \rightarrow \infty} \alpha^{(n)}=0, \lim _{n \rightarrow \infty} U^{(n)}=1, \lim _{n \rightarrow \infty} \Omega^{(n)}=0
$$

where we used the fact

$$
\lim _{n \rightarrow \infty}\left\{(1-U)_{n}-(1-U)_{n-1}\right\}=\epsilon
$$

for an infinitesimal constant $\epsilon(>0)$ accompanying with equation (27) to derive the above second and third limits as

$$
\begin{aligned}
& \lim _{n \rightarrow \infty} U^{(n)}=\lim _{n \rightarrow \infty} \frac{1-(1-U)_{n}}{1-(1-U)_{n-1}}=\lim _{n \rightarrow \infty} \frac{1-(1-U)_{n}}{1-(1-U)_{n}+\epsilon}=\frac{1-\alpha}{1-\alpha+\epsilon}=1, \\
& \lim _{n \rightarrow \infty} \Omega^{(n)}=\lim _{n \rightarrow \infty} \frac{\alpha-(1-U)_{n}}{\alpha-(1-U)_{n-1}}=\lim _{n \rightarrow \infty} \frac{\alpha-(1-U)_{n}}{\alpha-(1-U)_{n}+\epsilon}=\frac{0}{\epsilon}=0 .
\end{aligned}
$$

We next consider the case of $\alpha>1$. For this case, all students can get the job in the limit of $n \rightarrow \infty$. Hence, we have

$$
\lim _{n \rightarrow \infty}(1-U)_{n}=1 .
$$

By taking into account this fact, we immediately obtain

$$
\lim _{n \rightarrow \infty} \alpha^{(n)}=\infty, \lim _{n \rightarrow \infty} U^{(n)}=0, \lim _{n \rightarrow \infty} \Omega^{(n)}=1
$$

where we used the relation (29) and (32) to get the second and third limits as

$$
\begin{aligned}
& \lim _{n \rightarrow \infty} U^{(n)}=\lim _{n \rightarrow \infty} \frac{1-(1-U)_{n}}{1-(1-U)_{n}+\epsilon}=\frac{0}{\epsilon}=0 \\
& \lim _{n \rightarrow \infty} \Omega^{(n)}=\lim _{n \rightarrow \infty} \frac{\alpha-(1-U)_{n}}{\alpha-(1-U)_{n}+\epsilon}=\frac{\alpha-1}{\alpha-1+\epsilon}=1 .
\end{aligned}
$$




\subsubsection{The marginal $\alpha=1$ as a special case}

In the previous sections, we investigated the behavior of $\alpha^{(n)}$ for both cases of $\alpha>1$ and $\alpha<1$. Here we would like to stress that the marginal case $\alpha=1$ is rather special in the sense that $\alpha^{(n)}$ becomes independent of $n$ as

$$
\alpha^{(n)}=1 .
$$

This result is intuitively understood as follows. Let us define $\Delta_{V}^{(n)}$ and $\Delta_{N}^{(n)}$ as the number of positions which are occupied and the number of students who obtain the position at $n$-stage, respectively. Then, $\alpha^{(n)}$ is given as

$$
\alpha^{(n)} \equiv \frac{V^{(n)}}{N^{(n)}}=\frac{N-\Delta_{V}^{(1)}-\Delta_{V}^{(2)}-\cdots-\Delta_{V}^{(n-1)}}{N-\Delta_{N}^{(1)}-\Delta_{N}^{(2)}-\cdots-\Delta_{N}^{(n-1)}}=1
$$

where we used $V=\alpha N=N$. We also utilized the fact that the number of students who obtain the positions at each stage is identical to the number of positions occupied by those students, namely, $\Delta_{V}^{(k)}=\Delta_{N}^{(k)}, k=1, \cdots, n-1$.

From the result (25), we also confirm

$$
U^{(n)}=\Omega^{(n)}
$$

for $\alpha=1$. Thus, the performance of students to find the positions is identical to that of companies to fill the quota at each stage $n$.
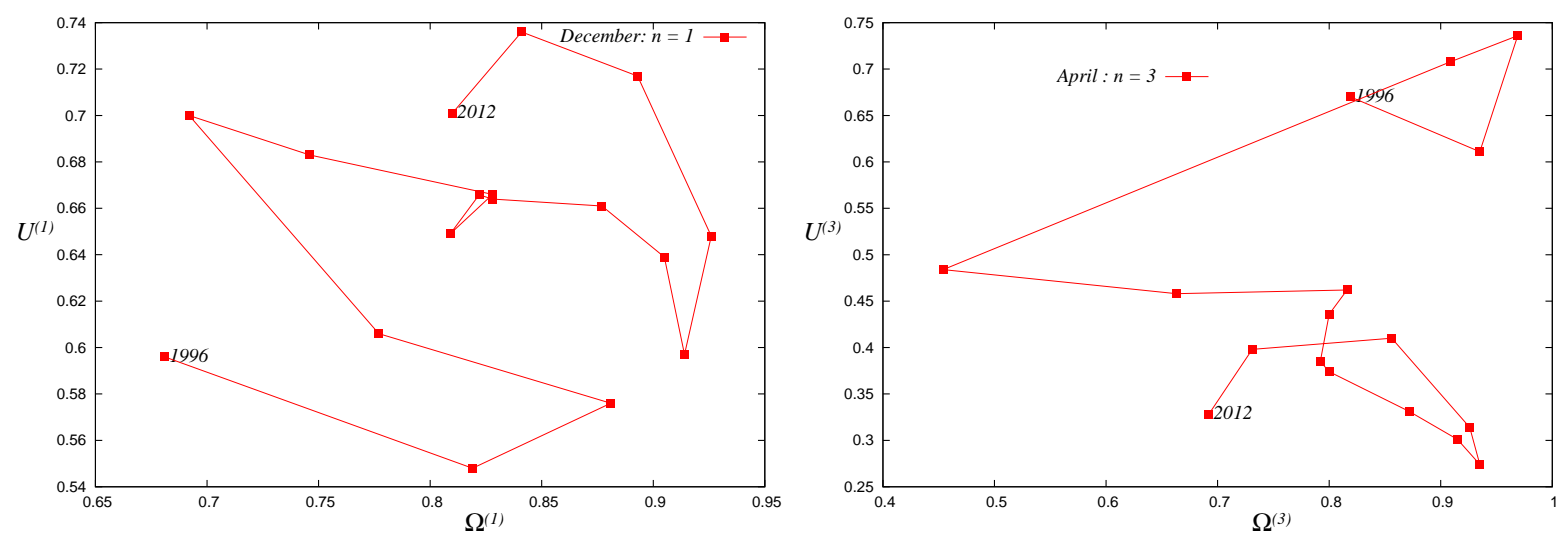

Figure 5: The $U-\Omega$ curves evaluated by means of stage-wise quantities. Namely, $U^{(1)}-\Omega^{(1)}$ curve (left) and $U^{(3)}-\Omega^{(3)}$ curve (right). The trajectories $\left(\Omega_{\text {year }}^{(n)}, U_{\text {year }}^{(n)}\right)$, year $=1996,1997, \cdots, 2012$ are calculated in terms of the cumulative employment rate $(1-U)_{n}$ by equation 25 .

In Figure 5, we plot the $U-\Omega$ curves evaluated by means of stage-wise quantities. Namely, $U^{(1)}-\Omega^{(1)}$ curve (left) and $U^{(3)}-\Omega^{(3)}$ curve (right). From the right panel accompanying with the upper left panel in Figure 3, which is the $U^{(0)}-\Omega^{(0)}$ curve from the definition, we clearly find that in 1996, the lowest global mismatch in $U^{(0)}-\Omega^{(0)}$ curve is getting worse as the stage $n$ gains up to $n=3$ as shown in Figure 5 (right). From the result, we are confirmed that the global mismatch could be changed as the stage $n$ goes on and it implies that students might change their strategies ('softening' their policies in some sense) during their job-hunting processes to get the positions.

\section{4 'Learning curve' for the collective intelligence}

In the previous sections, we derived the stage-wise quantities $\left(\alpha^{(n)}, U^{(n)}, \Omega^{(n)}\right)$ in terms of the cumulative employment rate $(1-U)_{n},(1-U)_{n-1}$ and $\alpha$. We also discussed several generic properties concerning these stage-wise quantities. However, we need a concrete 'learning algorithm' of collective intelligence which consists of job-seeking students. If these job-seeking students are 'intelligent' enough to obtain their positions as quick as possible. In other words, if the students' strategies are effective enough to fill the total vacancies at as early stage as possible, the 'learning algorithm' should be regarded as the best one. As the result, the quantity:

$$
\varepsilon_{n} \equiv \begin{cases}1-(1-U)_{n} & (\alpha \geq 1) \\ \alpha-(1-U)_{n} & (\alpha<1)\end{cases}
$$

which is regarded as an error-measurement for the collective intelligence, converges to zero at the earliest stage of $n$. Therefore, the $n$-dependence of the error-measurement $\varepsilon_{n}$ should be referred to as 'learning 
curve' (see e.g. Engle and Broeck (2001), Bishop (2006)], which is usually used in the literature of machine learning, for the behavior of collective intelligence of students.

Here we should mention that from equation (8), the cumulative labor shortage ratio $\Omega_{n}$ is a monotonically decreasing function of $n$ because $(1-U)_{n}$ increases monotonically. Therefore, the asymptotic limit is given by

$$
\lim _{n \rightarrow \infty} \Omega_{n}=\left\{\begin{array}{cc}
0 & (\alpha \leq 1) \\
\frac{\alpha-1}{\alpha} & (\alpha>1)
\end{array}\right.
$$

Obviously, we need to provide a specific learning algorithm to evaluate the learning curve. In following, we examine the learning curve for a simplest scenario, namely, the stage-wise unemployment rate $U^{(n)}$ or labor shortage ratio $\Omega^{(n)}$ is independent of $n$, namely, $U^{(n)}=U$ or $\Omega^{(n)}=\Omega$.

\subsubsection{A result from scale-invariance in $U$ or $\Omega$}

From equation (11), one can extract useful information about the $n$-dependence of the job-offer ratio on the condition that either $U$ or $\Omega$ is $n$-independent. We first consider the case in which the labor shortage ratio $\Omega$ is independent of $n$. By substituting (11) with a constant $\Omega$, that is, $U^{(k)}=\alpha^{(k)} \Omega+1-\alpha^{(k)}$ into equation (18), we have

$$
\alpha^{(n)}=\frac{\alpha-\left(1-\Omega^{n}\right) \prod_{k=0}^{n-1} \alpha^{(k)}}{1-\left(1-\Omega^{n}\right) \prod_{k=0}^{n-1} \alpha^{(k)}} .
$$

Solving the above equation with respect to $\alpha^{(n)}$, we have the solution as a function of constant $\Omega$ as

$$
\alpha^{(n)}=\frac{\alpha \Omega^{n}}{1-\alpha\left(1-\Omega^{n}\right)}, \quad \alpha<1
$$

where we should remember that we defined $\alpha \equiv \alpha^{(0)}$. Then, it should be stressed that the scale-invariant $\Omega$ is achieved if and only if the job-offer ratio $\alpha$ at the initial stage is smaller than unity. In other words, the scale-invariant $\Omega$ is never achieved for $\alpha>1$. From the general result we mentioned in the previous section, for the case of $\alpha<1$, the job-offer ratio $\alpha^{(n)}$ is a monotonically decreasing function of $n$. Therefore, we ultimately confirm that $\lim _{n \rightarrow \infty} \alpha^{(n)}=0$ and $\lim _{n \rightarrow \infty} U^{(n)}=1$. Namely, 'perfect unemployment state' could be realized at the end of our job-hunting process $n \rightarrow \infty$. In Figure 6 (left), we plot the $\alpha^{(n)}$ as a function of $n$ for the initial job-offer ratio $\alpha=0.5$ and 0.9 . The learning curve for this case is easily
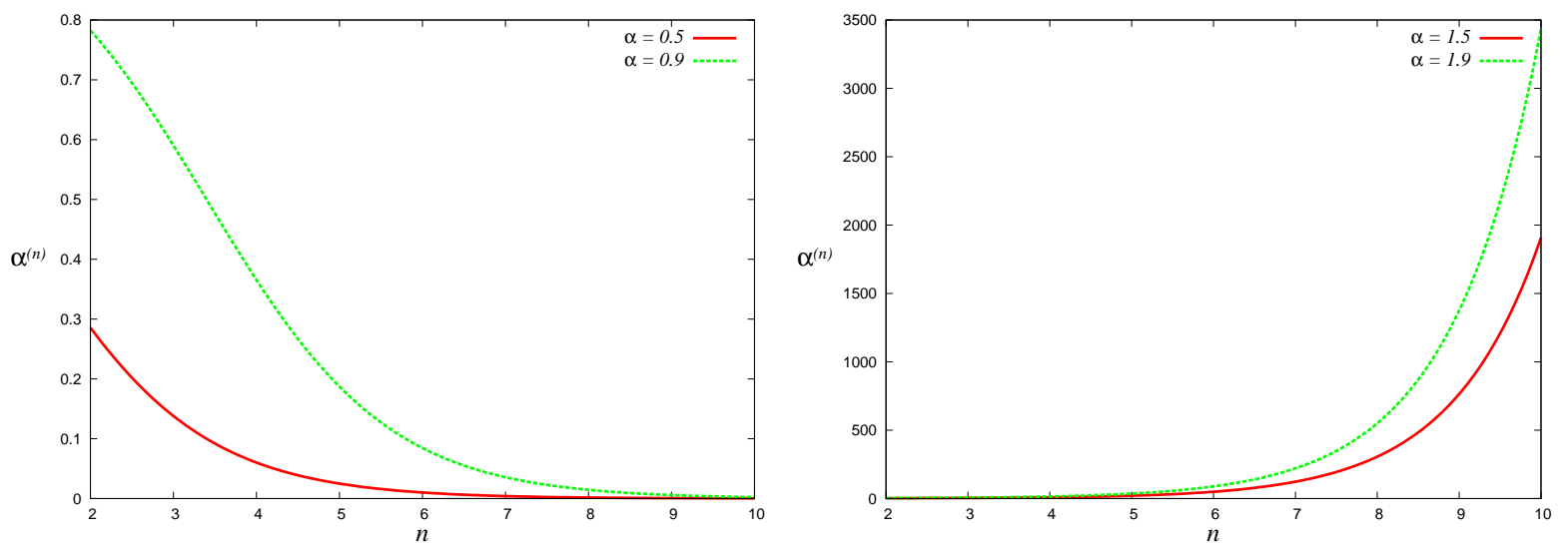

Figure 6: The $n$-dependence of job-offer ratio $\alpha^{(n)}$ for the case of $n$-invariant $\Omega=0.4$ (left) and $n$-invariant $U=0.4$ (right). The behavior of $\alpha^{(n)}$ is completely determined by the sign of $\alpha-1$ ( $\alpha=1$ is the marginal).

obtained as follows. Substituting (42) into (7), we have $U^{(k)}=\left\{1-\alpha\left(1-\Omega^{k+1}\right)\right\} /\left\{1-\alpha\left(1-\Omega^{k}\right)\right\}$. Hence, accompanying this result with $(1-U)_{n}=1-\prod_{k=0}^{n-1} U^{(k)}$, the error-measurement $\varepsilon_{n}$ defined by (39) for the case of $\alpha<1$ is now written as

$$
\begin{aligned}
\varepsilon_{n} & =\alpha-(1-U)_{n} \\
& =\alpha-\left\{1-\prod_{k=0}^{n-1} \frac{1-\alpha\left(1-\Omega^{k+1}\right)}{1-\alpha\left(1-\Omega^{k}\right)}\right\}=\alpha \Omega^{n}=\alpha \exp [-n \log (1 / \Omega)] .
\end{aligned}
$$

Therefore, the error-measurement $\varepsilon_{n}$ ('learning curve') decays exponentially to zero. In other words, the cumulative employment rate $(1-U)_{n}$ converges to the theoretical upper bound $\alpha(<1)$ with an exponential speed. 
obtain

On the other hand, for the case of $n$-independent $U$, by setting $U^{(k)}=U$ in (18), we immediately

$$
\alpha^{(n)}=\frac{\alpha-1+\prod_{k=0}^{n-1} U^{(k)}}{\prod_{k=0}^{n-1} U^{(k)}}=\frac{\alpha-1+U^{n}}{U^{n}}, \quad \alpha>1 .
$$

Here we should notice that the scale-invariant $U$ is achieved if and only if $\alpha$ satisfies $\alpha>1$. Then, the $\alpha^{(n)}$ is a monotonically increasing function of $n$ and it reads $\lim _{n \rightarrow \infty} \alpha^{(n)}=\infty$ and $\lim _{n \rightarrow \infty} \Omega^{(n)}=1$. This means that 'perfect labor shortage state' appears at the final stage of our job-hunting process. When we use the error measurement in the collective behavior of students from (39) as

$$
\varepsilon_{n} \equiv 1-(1-U)_{n}
$$

for $\alpha>1$, the $\varepsilon_{n}$ as a function of $n$ could be regarded as a learning curve for this case. For the scale-invariant $U$, the curve decays to zero exponentially as

$$
\varepsilon_{n}=U^{n}=\exp [-n \log (1 / U)]
$$

Therefore, the error-measurement decays exponentially to zero, in other words, the cumulative employment rate $(1-U)_{n}$ converges to unity, which means that all students can obtain the position asymptotically.

The above two limiting cases seem to be rather special. Thus, one can naturally assume that both $\Omega$ and $U$ could be dependent on the job-hunting stage $n$. In order to consider such general case somehow or other, we shall revisit our probabilistic labor market Chen and Inoue (2012a), Chen and Inoue (2012b)] Of course, in macroeconomics (labor science), there exist a lot of effective attempts to discuss the macroscopic properties Gabriele (2002), Fagiolo, Dosi and Gabriele (2004), Neugart (2004)] including so-called search theory Lippman and McCall (1976), Diamond (1982), Pissarides (1985), Pissarides (2000)]. However, it should be stressed that the macroscopic approaches lack of their microscopic viewpoint, namely, in their arguments, the behaviour of microscopic (heterogeneous) agents such as job-seekers or companies are neglected.

\section{The model system}

In this section, in order to derive the annual $U-\Omega$ curve that we have shown in the previous section or or $n$-dependence of the macroscopic quantities for general scenario, we will briefly review our model system that we have defined in previous studies Chen and Inoue (2012a), Chen and Inoue (2012b).

\subsection{Assumptions}

We first introduce several basic properties which should be satisfied in our probabilistic labor market. In our model system, the labors (the job seekers) are restricted to university graduates and the other people on the job searching are neglected for simplicity. In our modeling, we shall assume that the following four simple points (i)-(iv) should be taken into account to construct the labor markets.

(i) Each company recruits constant numbers of newcomers $v_{k}^{*} \in \mathbb{Z}$ in each year.

(ii) If the company takes too much or too less applications which are far beyond or far below the quota, the ability of the company to gather applicants in the next business year decreases.

(iii) Each company is apparently ranked according to various perspectives, and it is available for all students.

(iv) A diversity of making the decision of labor should be taken into account by means of maximization of Shannon's entropy under some relevant constraints.

\subsubsection{Local mismatch measurement}

Considering the condition (ii) cited above, we shall define the following local mismatch measurement:

$$
h_{k}(t)=\frac{1}{V}\left|v_{k}^{*}-v_{k}(t)\right|=\frac{1}{\alpha N}\left|v_{k}^{*}-v_{k}(t)\right|
$$

where $v_{k}(t)$ denotes the number of students who seek for the position in the company $k$ at the business year $t$. Hence, the above local mismatch measurement $h_{k}(t)$ stands for the difference (gap) between the number of applicants $v_{k}(t)$ and the quota $v_{k}^{*}$ for each company $k$. 


\subsubsection{Ranking factor}

On the other hand, based on the condition (iii), we define the ranking of the company $k$ by $\epsilon_{k}(>1)$ which is independent of the business year $t$. Here we assume that the ranking of the company $k$ is higher if the value of $\epsilon_{k}$ is larger. In our agent-based modeling, we simply set the value as

$$
\epsilon_{k}=1+\frac{k}{K} .
$$

In such a situation, the company $k=K$ is the highest ranking company and the company $k=1$ is the lowest. We should mention that in the second term in the right hand side of (48), we divided the $k$ by the number of companies $K$ so as to make the maximum ranking factor $\max _{k} \epsilon_{k}=\epsilon_{K}=2$ a size-independent quantity.

\subsubsection{Energy function: Student's preference of market history or ranking}

With the above definitions, the energy function of our probabilistic labor market for each company $k$ is written explicitly by

$$
E\left(\epsilon_{k}, h_{k} ; t\right) \equiv-\gamma \log \epsilon_{k}+\sum_{l=1}^{\tau} \beta_{k}(t-l) h_{k}(t-l) .
$$

When we define two $\tau$-dimensional market history vectors: $\boldsymbol{\beta}_{k} \equiv\left(\beta_{k}(t-1), \cdots, \beta_{k}(t-\tau)\right)$ and $\boldsymbol{h}_{k} \equiv$ $\left(h_{k}(t-1), \cdots, h_{k}(t-\tau)\right)$, the second term appearing in the above energy function (49) is defined by the inner product of these two vectors as $\left(\boldsymbol{\beta}_{k} \cdot \boldsymbol{h}_{k}\right)$. In this paper, we shall simply choose a particular market history vector as $\boldsymbol{\beta}_{k}=(\beta, 0, \cdots, 0)$ for all $k$.

Based on this energy function, the behavior of students can be considered as follows. From the first term of the energy function, students tend to post their applications to companies with relatively high ranking. However, from the second term appearing in the energy function, we should notice that even if the company $k$ gathers a lot of applicants as over the quota at some year $t$, the probability that the company $k$ gathers the applicants at the next business year $t+1$ decreases. Therefore, the second term depends on the history of the labor market as a 'negative feedback' on the first term. The information about the market in the previous business year $h_{k}(t)$ is much more important for the students for $\gamma>\beta$, whereas the ranking of companies becomes more essential for $\gamma<\beta$ to decrease the energy function.

Here we would like to mention the relationship between these parameter set $(\gamma, \beta)$ and heterogeneity in our labor market. Obviously, the preferences of companies' ranking and market history depend on a private individual because each students possess his/her own strategy to obtain the suitable position. In this sense, these parameters should be selected as $\left(\gamma_{i}, \beta_{i}\right)$ for $i=1, \cdots, N$. However, this type of choice might cause tremendous difficulties in our modeling because we should calibrate $2 N$ parameters from the empirical evidence. Of course, one can assume that $\gamma$ and $\beta$ are generated by some parametric distributions $P(\gamma)$ and $P(\beta)$ which contain a finite number of parameters. However, for this choice also, we need to determine the parameters from limited empirical data and it is not so easy for us to carry out. Therefore, in this paper, we simply assume that the parameters are independent of student. As we see in the next section, heterogeneous properties (diversities) in our model system are induced by means of the Jaynes-Shannon's MaxEnt principle.

\subsection{Jaynes-Shannon's MaxEnt principle}

In the previous sections, we introduced the energy function (49) to be minimized for each student to choose the company to apply for. However, minimizing the energy function leads to a unique solution without any diversity. The lack of diversity for the students' choices sometimes causes the tremendous global mismatch. In order to introduce the diversity into the system, we utilize the so-called JaynesShannon's MaxEnt principle [Jaynes (1957)]. In following, we derive the aggregation probability for the students by means of the maximum entropy principle.

As we mentioned, the decision-makings of labors that make up the labor market should be 'diverse' from the assumption (iv). In order to quantify the diversity, we maximize the following Shannon's entropy:

$$
H=-\sum_{k=1}^{K} P_{k}(t) \log P_{k}(t)
$$

under the normalization condition of the probability $P_{k}(t)$, namely,

$$
\sum_{k=1}^{K} P_{k}(t)=1
$$


and the conservation of energy

$$
E=\sum_{k=1}^{K} P_{k}(t) E\left(\epsilon_{k}, h_{k} ; t\right)
$$

in the each time (annual). Then, we consider the following variational problem described by the functional Jaynes (1957)] concerning $P_{k}(t)$ :

$$
f\left[P_{k}(t)\right]=-\sum_{k=1}^{K} P_{k}(t) \log P_{k}(t)+\lambda_{1}\left\{1-\sum_{k=1}^{K} P_{k}(t)\right\}+\lambda_{2}\left\{E-\sum_{k=1}^{K} P_{k}(t) E\left(\epsilon_{k}, h_{k} ; t\right)\right\}
$$

where $\lambda_{1}$ and $\lambda_{2}$ are Lagrange multipliers. By solving this variational problem with respect to $P_{k}(t)$ so as to satisfy

$$
\frac{\delta f\left[P_{k}(t)\right]}{\delta P_{k}(t)}=\frac{\delta f\left[P_{k}(t)\right]}{\delta \lambda_{1}}=\frac{\delta f\left[P_{k}(t)\right]}{\delta \lambda_{2}}=0,
$$

the probability $P_{k}(t)$ that the company $k$ gathers the candidates at time $t$ is given by

$$
P_{k}(t)=\frac{\exp \left[\gamma \log \left(1+\frac{k}{K}\right)-\beta\left|v_{k}^{*}-v_{k}(t-1)\right|\right]}{\sum_{k=1}^{K} \exp \left[\gamma \log \left(1+\frac{k}{K}\right)-\beta\left|v_{k}^{*}-v_{k}(t-1)\right|\right]}
$$

where we chose $\mathrm{e}^{-1-\lambda_{1}}=\left\{\sum_{k=1}^{K} \exp \left[\gamma \log \left(1+\frac{k}{K}\right)-\beta\left|v_{k}^{*}-v_{k}(t-1)\right|\right]\right\}^{-1}$ and set $\lambda_{2}=1$ (unit 'temperature' to control the diversity of the system) for simplicity. Therefore, for each time step $t$, each student posts his/her application letter to the company $k$ according to the above aggregation probability (55).

It is worth while for us to mention that the probability described by (55) has a quite similar formula to the so-called multinomial (non-linear) logit model or softmax regression in the literature of statistics (see e.g. McFadden (1973), Train (2003)]). Actually, when we define the so-called softmax function as

$$
\operatorname{softmax}\left(k, x_{1}, x_{2}, \cdots, x_{K}\right)=\frac{\exp \left(x_{k}\right)}{\sum_{i=1}^{K} \exp \left(x_{i}\right)},
$$

the aggregation probability $P_{k}(t)$ is written by

$$
P_{k}(t)=\operatorname{softmax}\left(k,-E_{1}(t),-E_{2}(t), \cdots,-E_{K}(t)\right) .
$$

Henceforth, the actions of students to post their application letters are governed by the aggregation probability (55). To monitor their 'microscopic' actions, we introduce several microscopic and macroscopic quantities in the following sections.

\subsubsection{Microscopic variables}

Here we define the microscopic variable $s_{i k}(t)$ which is used to distinguish whether the student $i$ receives an acceptance from the company $k$ in the business year $t$ or not. Namely, $s_{i k}(t)=1$ means that the student $i$ receives an acceptance from the company $k$ in the business year $t$, and $s_{i k}(t)=0$ means that he/she does not. In particular, the microscopic variable $a_{i k}(t)$ is used to distinguish whether the student $i$ decides to post his/her application letter to the company $k$ at time $t$ or not as

$$
a_{i k}(t)= \begin{cases}1 & \left(\text { with } P_{k}(t)\right) \\ 0 & \left(\text { with } 1-P_{k}(t)\right)\end{cases}
$$

Then, we should notice that the total number of acceptances for the student $i$ is defined by

$$
s_{i}(t)=\sum_{k=1}^{K} s_{i k}(t)
$$

with

$$
P\left(s_{i k}(t)=1 \mid a_{i k}(t)\right)=\Theta\left(v_{k}^{*}-v_{k}(t)\right) \delta_{a_{i k}(t), 1}+\frac{v_{k}^{*}}{v_{k}(t)} \Theta\left(v_{k}(t)-v_{k}^{*}\right) \delta_{a_{i k}(t), 1}
$$

and $P\left(s_{i k}(t)=0 \mid a_{i k}(t)\right)=1-P\left(s_{i k}(t)=1 \mid a_{i k}(t)\right)$, where $\delta_{x, y}$ stands for the Kronecker's delta, and $\Theta(x)$ denotes the conventional step function defined by

$$
\Theta(x)= \begin{cases}1 & (x \geq 1) \\ 0 & (x<0)\end{cases}
$$


Thus, the first term of equation (60) means that the $s_{i k}(t)$ takes 1 with unit probability when the student $i$ posts the application to the company $k$ and the total number of applications the company $k$ gathered does not exceed the quota $v_{k}^{*}$. On the other hand, the second term means that the $s_{i k}(t)$ takes 1 with probability $v_{k}^{*} / v_{k}(t)$ even if $v_{k}(t)>v_{k}^{*}$ holds. In other words, for $v_{k}(t)>v_{k}^{*}$, the informally accepted $v_{k}^{*}$ students are randomly selected from $v_{k}(t)$ candidates.

In this paper, we use the above 'random selection' by companies, however, it is possible for us to extend the selection process by companies to much more realistic version. For instance, in the reference Chen and Inoue (2013), we considered a scenario in which each company that obtained application letters over the quota selects the candidates according to their scores (school records).

\section{The results}

In this section, we carried out agent-based simulations based on our probabilistic model described by (55) (60) and (58). Before we simulate the artificial labor market, we should determine (estimate) several parameters such as the degree of ranking preference $\gamma$, market history preference $\beta$ and average number of application letters $a$ from the available empirical data.

\subsection{Model calibration and theoretical $U-\Omega$ curve}

To determine the parameters appearing in the system, we focus on the following unemployment rate as an order parameters.

$$
U \equiv U_{0}=U^{(0)}=\lim _{T \rightarrow \infty} \frac{1}{T} \sum_{t=0}^{T-1} U_{t}, \quad U_{t}=\frac{1}{N} \sum_{i=1}^{N} \delta_{s_{i}(t), 0} .
$$

The labor shortage ratio $\Omega \equiv \Omega_{0}=\Omega^{(0)}$ is evaluated by inserting the above result (62) into equation (11). As we mentioned, in order to draw the $U-\Omega$ curve, we need information about the system parameters such as strength of ranking preference $\gamma$ and market history $\beta$. Here we fix $\beta=1$ and look for the best possible $\gamma$ as a solution of $U_{\text {empirical }}=U(\gamma)$, namely,

$$
\gamma=U^{-1}\left(U_{\text {empirical }}\right)
$$

where $U_{\text {empirical }}$ denotes the empirical unemployment rate for a given job-offer ratio $\alpha \equiv \alpha^{(0)}$, which is opened for the public by MEXT \& MHLW [MEXT] in every October (see Figure 1(left)). In the reference Chen and Inoue (2012a), Chen and Inoue (2012b)], we showed that the cumulative employment rate $1-U=(1-U)_{0}$ monotonically decreases as $\gamma$ increases. It means that there exists a unique solution of (63) for a given empirical data $U_{\text {empirical }}$. In our simulations, we set $N=2000, K=100$ and $n_{k}^{*}=\eta, \forall_{k}$, and it turns out to be

$$
\eta=\frac{N}{K} \alpha=20 \alpha
$$

for a given $\alpha$. Hence, we can control the $\eta$ to realize any positive value of $\alpha$.

We plot the resulting $\gamma$ and $U-\Omega$ curve in Figure 7 . From this figure, we find that the large $\gamma$ apparently pushes the $U-\Omega$ curve toward the upper right direction where the global mismatch between the students and companies is extremely large. The gap between the theoretical and empirical $U-\Omega$ curve comes from the uncertainties in the calibration of average number of application letters $a$. Obviously, it is observable quantity and a large scale survey tells us the precise statistics. However, up to know, there is no authorized reliable statistics although several private researches provided the data. Hence, we simply chose the value as $a=10$ in our simulation.

We should bear in mind that the parameter $\gamma$ is not observable in principle. However, our approach enables us to mention the annual strength of students' ranking preferences $\gamma$ against that of the market history $\beta$. In 2010 when the global mismatch was the largest, the point $(\Omega, U)$ is located at the upper right position in the $U-\Omega$ curve. However, the corresponding $\gamma$ does not take the peak in the annual $\gamma$ trends and the peak appeared in 2008. At a glance, it seems to be inconsistent, however from Figure 1 (right), we find that the job-offer ratio $\alpha$ in 2010 is larger than that in 2008. This implies that the market in 2010 was more 'seller's market' than in 2008, and the market supplied a lot of positions to job-seeking students. Actually, unemployment rate $U$ in 2008 was relatively low (see Figure 1 (left)). On the other hand, in 2010, the unemployment rate increases by 0.6 from 2009 due to the decreases of $\alpha$ although the strength of students' ranking preferences was getting smaller during 2009-2010. As the result, the point $(\Omega, U)$ moved toward the upper right position in the $U-\Omega$ curve (see Figure 7 (right)), which resulted in the worst global mismatch between students and companies in 2010. 

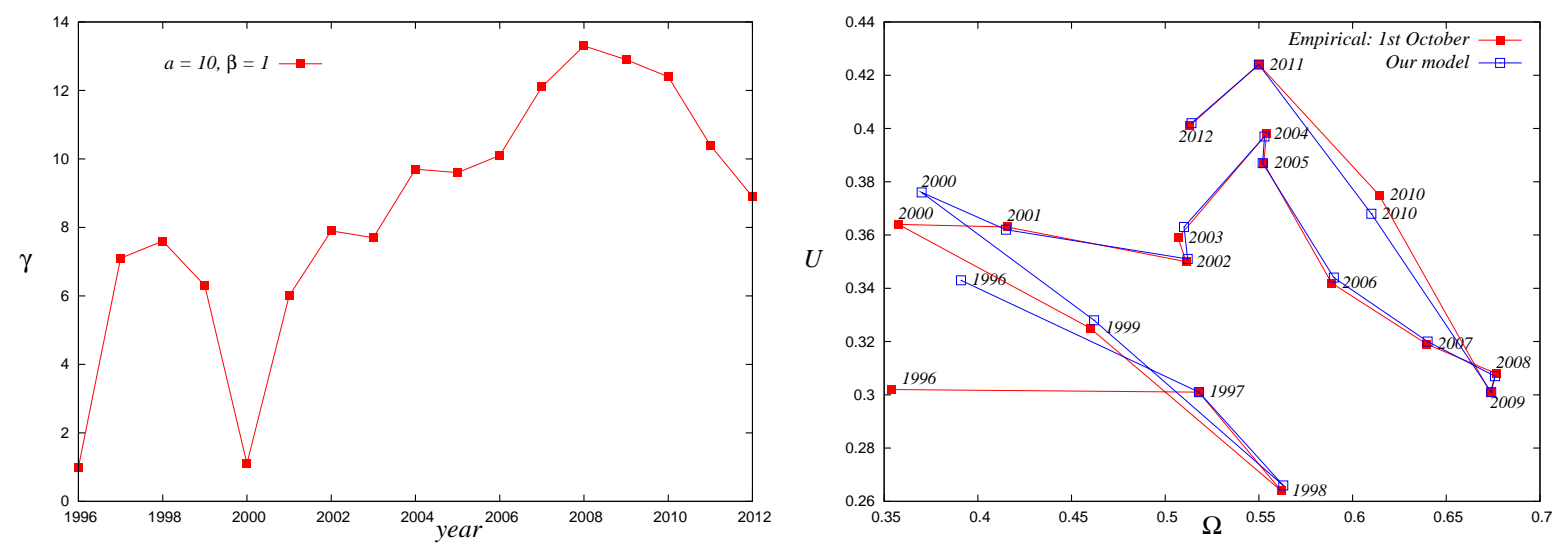

Figure 7: Time dependence of ranking preference $\gamma$ (left). By using this panel accompanying with the right panel in Figure 1 (right), one can account for the appearance of global mismatch. The right panel shows the empirical and theoretical $U-\Omega$ curves. We clearly find that the large $\gamma$ apparently pushes the $U-\Omega$ curve toward the upper right direction where the global mismatch between the students and companies is large.

\subsection{Analysis for the general case}

As we already mentioned before, both $U$ and $\Omega$ are not scale-invariant in general. Therefore, we should investigate the flow of relevant quantities $\left(\alpha^{(n)}, U^{(n)}, \Omega^{(n)}\right)$ by means of our probabilistic labor market Chen and Inoue (2012a), Chen and Inoue (2012b)]. After several complicated algebra, we obtain the following coupled 'dynamical' equations.

$$
\begin{aligned}
P_{k}(t) & =\frac{\exp \left[\gamma \log \left(1+\frac{k}{K}\right)-\beta\left|v_{k}^{*}-v_{k}(t-1)\right|\right]}{\sum_{k=1}^{K} \exp \left[\gamma \log \left(1+\frac{k}{K}\right)-\beta\left|v_{k}^{*}-v_{k}(t-1)\right|\right]} \\
\alpha^{(t)} & =\frac{\sum_{k=1}^{K}\left(v_{k}^{*}(t)-m_{k}(t)\right) \delta_{v_{k}^{*}(t), m_{k}(t)}}{\sum_{i=1}^{N} \prod_{l \leq t} \delta_{s_{i}(l), 0}} \\
U^{(t)} & =1-\frac{\sum_{i=1}^{N} \prod_{l \leq t} \delta_{s_{i}(l), 0}}{\sum_{i=1}^{N} \prod_{l \leq t-1} \delta_{s_{i}(l-1), 0}} \\
\Omega^{(t)} & =\frac{U^{(t)}+\alpha^{(t-1)}-1}{\alpha^{(t-1)}} \\
(1-U)_{n} & =\frac{\sum_{i=1}^{N} \prod_{l \leq t} \delta_{s_{i}(l), 0}}{N}
\end{aligned}
$$

We carry out computer simulations based on the above equations (65)-(69).

\subsubsection{Collective behavior of zero-intelligence students}

In our simulation, we calibrate the parameters $\gamma, \beta$ by making use of the empirical data which is opened to the public in every October. After the calibration, we do not change the variables. In this sense, the students do not 'learn' at all from the past markets (stages). In real labor market for university graduates, students who failed to get the position at $n=0$ might change their strategies. For instance, they might decreases the strength of ranking preference $\gamma$ to look for much broadly including small enterprises. However, we do not take into account such 'intelligence' of the students and we investigate to what extent the collective behaviour of such 'zero-intelligence' students can reduce the global mismatch.

In Figure 8, we plot $\alpha^{(n)}$ and $1-U^{(n)}, \Omega^{(n)}$ in the last three years, and the cumulative employment rate $(1-U)_{n}$ as a function of $n$ in 2012. From these panels, we clearly find that the relevant quantities $\left(\alpha^{(n)}, U^{(n)}, \Omega^{(n)}\right)$ are not scale-invariant at all and each of them possesses a rather complicated $n$-dependence. It should be stressed that the empirical data set for $\left(\alpha^{(n)}, U^{(n)}, \Omega^{(n)}\right)$ is not available (has never been released to the public) and we just have the data for the cumulative employment rate $(1-U)_{n}$ instead of $U^{(n)}$ as shown in Figure 1. In this sense, our result might be addressed as the 'first attempt' to show the stage $n$-dependence of the quantities $\left(\alpha^{(n)}, U^{(n)}, \Omega^{(n)}\right)$ explicitly in Japanese labor market.

In the lower right panel, we show the cumulative employment rate $(1-U)_{n}$ with the counter empirical plot. We are confirmed that the theoretical result is remarkably improved from the simple observation $(1-U)_{n}=1-U^{n}$ as shown in Figure 1 (right). However, there still exists a gap between empirical 

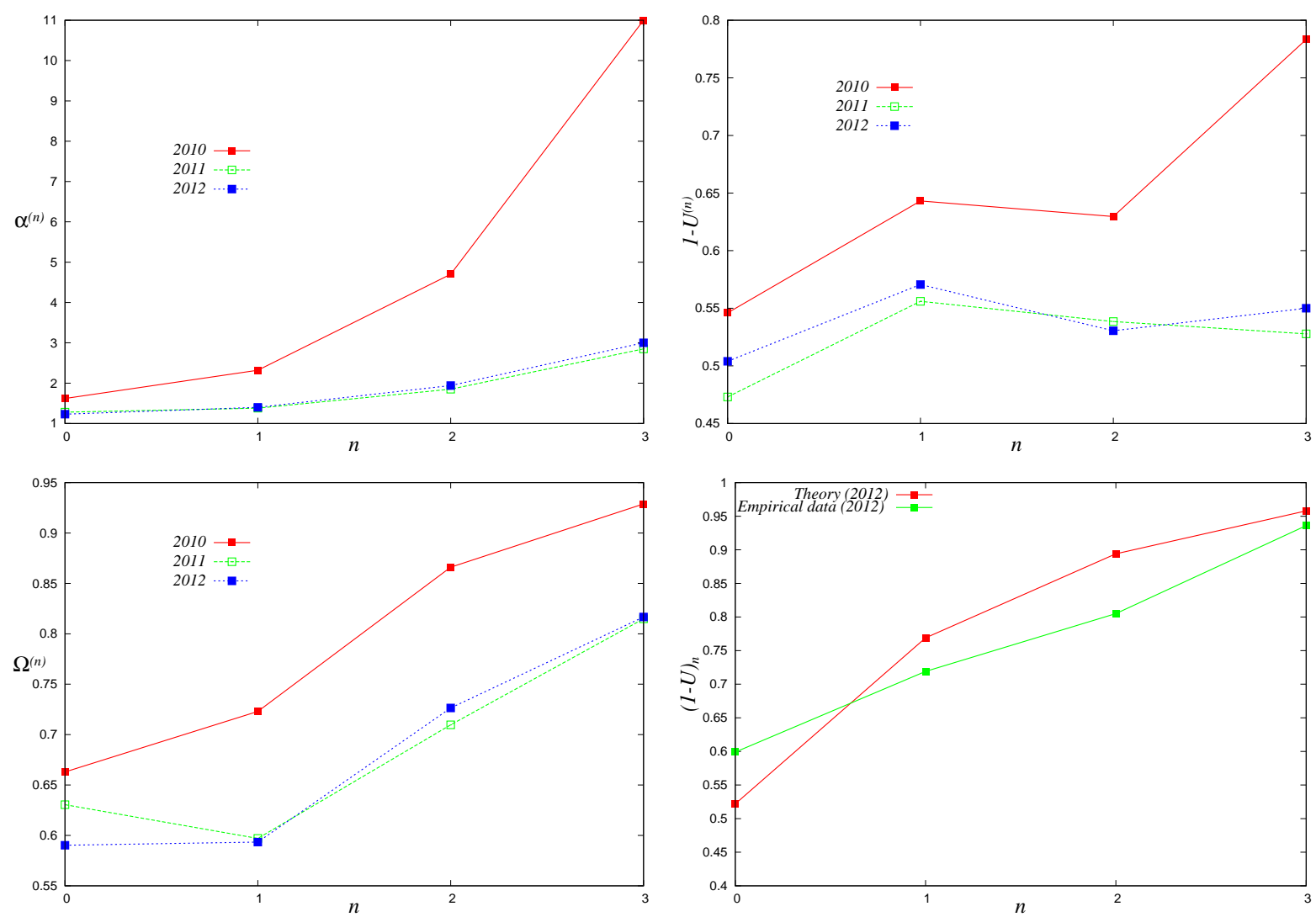

Figure 8: From the upper left to the lower right, $\alpha^{(n)}$ and $1-U^{(n)}, \Omega^{(n)}$ in the last three years, and the cumulative employment rate $(1-U)_{n}$ as a function of $n$ in 2012 are shown.

and theoretical results. The gap might come from wrong calibration of system parameters such as the average number of application letters $a$ for a student etc. (In the above simulations, we set $a=10$ ).

Finally, in Figure 9, we show the learning curves as the cumulative employment rate $(1-U)_{n}$ for the case of $a=5$ (left) and $a=10$ (right). In these panels, we examine for $\alpha=0.5$ and 2. From this figure, we clearly observe that the $(1-U)_{n}$ converges to 1 for $\alpha=2(>1)$ and $\alpha$ for $\alpha=0.5(<1)$ as we have discussed in the previous sections. Here we should focus on the speed of convergence. From both panels,
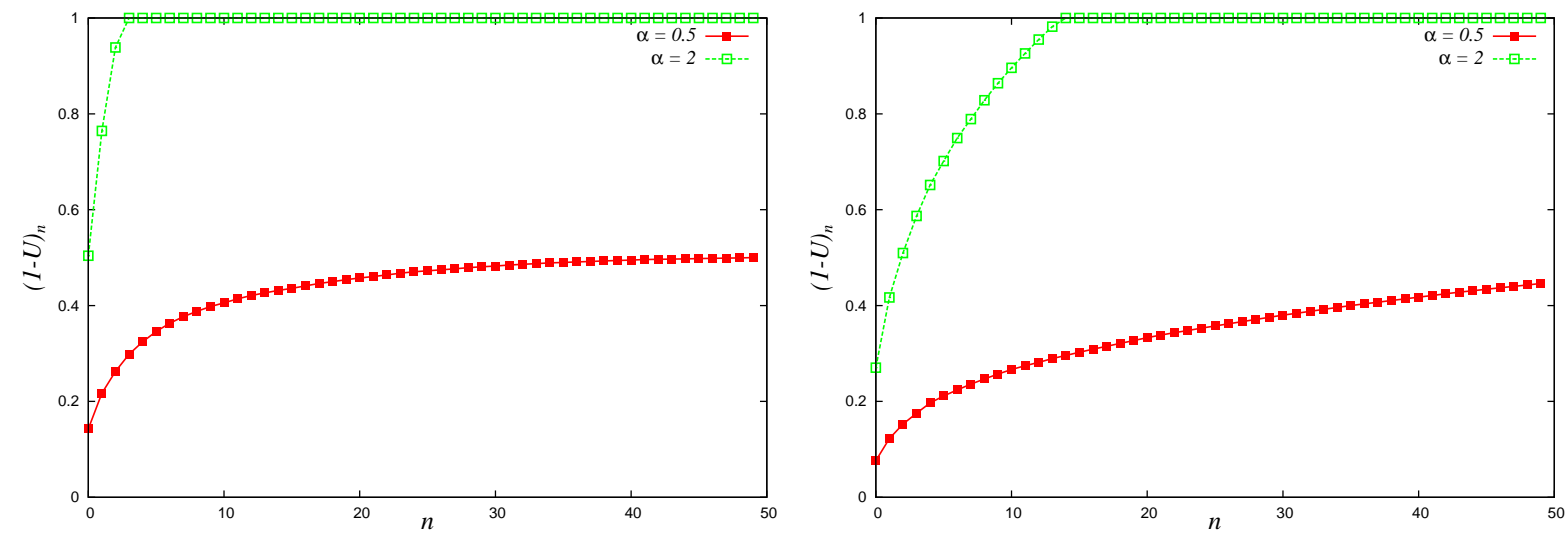

Figure 9: The learning curves as the cumulative employment rate $(1-U)_{n}$ for the case of $a=5$ (left) and $a=10$ (right). We set the system parameters simply as $\gamma=\beta=1$.

we find that the speed of the convergence of $(1-U)_{n}$ to the theoretical upper bound unity for the case of $\alpha>1$ is much faster than that of the case for $\alpha<1$ (the theoretical upper bound is $\alpha$ ). This fact might imply that the job-matching between students and companies in 'buyer's market' is much harder than that in 'seller's market' especially in the large $n$ regime. It is quite reasonable because $\alpha^{(n)}$ decays to zero for $\alpha<1$. Another remarkable finding is about average number of application letters, and actually we find that the increase of $a$ causes tremendous speed down in the convergence. 


\section{Summary}

Inspired by the unsupervised learning or self-organization in the machine learning context, we drew 'learning curve' for the collective behavior of job-seeking 'zero-intelligence' labors in successive job-hunting process. We discussed the scale invariance of the macroscopic quantities in probabilistic labor markets. We obtained the flow $\left(\alpha^{(n)}, U^{(n)}, \Omega^{(n)}\right)$ with the assistance of computer simulations. Obviously, students in real labor markets are much more 'intelligent' than the agents in our model system. In this sense, we should modify our model by taking into account the students' tendency of job-hunting process. Especially, 'intelligent' learning algorithm of system parameters such as $\gamma$ or $\beta$ might play important role in the next direction of our studies. These parameters might be dependent on the business cycle in the society and in this sense, the labor market should be coupled with the other kinds of markets such as financial market or housing market. These issues should be addressed as our future problems.

\section{Acknowledgement}

This work was financially supported by Grant-in-Aid for Scientific Research (C) of Japan Society for the Promotion of Science, No. 22500195. We acknowledge Recruit Works Institute for allowing us to use their very recent survey data [Recruit Works Institute (2013)]. One of the authors (JI) thanks Yuji Aruka, Yoshi Fujiwara and Hideaki Aoyama for fruitful discussion and for encouraging us to submit our new result to Evolutionary and Institutional Economics Review.

\section{References}

[Aoki and Yoshikawa (2006)] Aoki M and Yoshikawa H (2006) Reconstructing Macroeconomics: A Perspective from Statistical Physics and Combinatorial Stochastic Processes, Cambridge University Press.

[Bishop (2006)] Bishop C M (2006) Pattern Recognition and Machine Learning, Springer.

[Boeri and Ours (2008)] Boeri T and Ours J van (2008) The Economics of Imperfect Labor Markets, Princeton University Press.

[Chen and Inoue (2012a)] Chen H and Inoue J (2012) "Dynamics of probabilistic labor markets: statistical physics perspective", Lecture Notes in Economics and Mathematical Systems 662, pp. 53-64, "Managing Market Complexity", Springer.

[Chen and Inoue (2012b)] Chen H and Inoue J (2012) "Statistical Mechanics of Labor Markets, Econophysics of systemic risk and network dynamics", New Economic Windows 2013, Springer-Verlag (Milan-Italy), pp.157-171.

[Chen and Inoue (2013)] H. Chen and J. Inoue, in preparation.

[Diamond (1982)] Diamond P A (1982) "Aggregate Demand Management in Search Equilibrium", Journal of Political Economy 90, pp. 881-894.

[Engle and Broeck (2001)] Engle A and Broeck V. den (2001) Statistical Mechanics of Learning, Cambridge University Press.

[Fagiolo, Dosi and Gabriele (2004)] Fagiolo G, Dosi G and Gabriele R (2004) "Matching, Bargaining, and wage setting in an evolutionary model of labor market and output dynamics", Advances in Complex System 7, No.2, pp. 157-186.

[Gabriele (2002)] Gabriele R (2002) "Labor Market Dynamics and Institution: An Evolutionary Approach", Working Paper in Laboratory of Economics and Management Sant'Anna School of Advances Studies, Pisa, Italy.

[Hino and Inoue (2011)] Hino H and Inoue J (2011) "Reconstructing Philips curves in macroeconomics from the microscopic description of stochastic labor markets", in Proceedings of Markov and Semi-Markov Processes \& Related Fields, CD-ROM. See also Hino H (2011) "Reconstruction of Philips curves in 'macro'economics from the 'micro'scopic description of artificial labor markets", Master Thesis (Hokkaido University, 2011): http://eprints.lib.hokudai.ac.jp/dspace/handle/2115/44985 (in Japanese). 
[Jaynes (1957)] Jaynes E T (1957) "Information Theory and Statistical Mechanics", Physical Review 106, pp. 620-630.

[Lippman and McCall (1976)] Lippman S and McCall J J (1976) "The Economics of Job Search: A Survey", Economic Inquiry 14, pp. 155-188.

[McFadden (1973)] McFadden D (1973) "Conditional logit analysis of qualitative choice behavior" In Frontiers in Econometrics, Academic Press, New York.

[METI] http://www.meti.go.jp/statistics/tyo/kikatu/result-2/h21kakuho.html

[MEXT] http://www.mext.go.jp, http://www.mhlw.go.jp

[MHLW] http://www.mhlw.go.jp/stf/houdou/2r98520000006hma.html

[Neugart (2004)] Neugart M (2004) "Complicated dynamics in a flow model of the labor market", Journal of Economic Behavior and Optimization 53, pp. 193-213.

[Philips (1958)] Philips A W (1958) "The Relation Between Unemployment and the Rate of Change of Money Wage Rates in the United Kingdom, 1861-1957", Economica, New Series 25, No. 100, pp. 283-299.

[Pissarides (1985)] Pissarides C A (1985) "Short-Run Equilibrium Dynamics of Unemployment Vacancies", American Economic Review 75, pp. 676-690.

[Pissarides (2000)] Pissarides C A (2000) Equilibrium Unemployment Theory, MIT Press.

[Recruit Works Institute] http://www.works-i.com/surveys/adoptiontrend/

[Recruit Works Institute (2013)] Recruit Works Institute (2013) "College Graduates Job Opening Survey", No. 30, April (in Japanese).

[Train (2003)] Train K (2003) Discrete choice methods with simulation, Cambridge University Press, New York. 